\title{
Research on the Grey Correlation Analysis of the Norm in Choosing Key Factors of Aero-engine Design Integration System
}

\author{
Ying $\mathrm{He}^{1,2}$ and Rui Xiu ${ }^{3}$ \\ ${ }^{1}$ School of mechanical engineering and automation, Beihang University, Beijing, China \\ ${ }^{2}$ AECC Hunan Aviation Power Plant Research Institute, Zhuzhou, China \\ ${ }^{3}$ Beijing Institute of Aerospace Control Instrument, Beijing, China
}

\begin{abstract}
The key influencing factor of accurate positioning aerospace design integration system is the basis of successful construction of design architecture integration platform. Normal grey relational analysis has better approximation than general grey relational analysis. The article analyses the effects of grey correlation analysis and aero-engine design integration system, and then based on $t$ norm grey correlation analysis, determine the key influence factors of aerospace design integration system, a more true and accurate result is obtained.
\end{abstract}

\section{Introduction}

The establishment of an aero-engine design integration system is a system design process. Systems design is the process of defining the architecture, modules, interfaces, and data for a system to satisfy specified requirements. Systems design could be seen as the application of systems theory to product development. There is some overlap with the disciplines of systems analysis, systems architecture and systems engineering. The relationship between the three is not described in the article.

The development of aero engine needs to integrate multiple professional work, and it has gone through several design stages that may iterate repeatedly, including demand analysis, overall scheme design, subsystem/component principle design, detailed design, virtual prototype analysis and simulation based on detailed design model. Data, processes, algorithms, knowledge and tools in the development of aero-engine need a unified platform for management, which also provides an opportunity for the development of aeroengine design integration system. Which of the functions of aero-engine design integration system are the important influence factors, the precious researches on identify the critical factors mainly focus on qualitative analysis, adopting methods such as experts' investigation and circular arch balance method [1], etc. In recent years, the quantitative research concerning the choosing key factors are on a rise. Among which, more frequently used methods are priority-degree evaluation [2]-[3] and fuzzy comprehensive evaluation [4]. The method of prioritydegree evaluation is an important evaluation method of the extension theory. It could consider both the qualitative and quantitative indexes that have influence on the subject investigated into the process of confirming the ultimate target. Fuzzy comprehensive evaluation could quantify subjective factors to evaluate things when information is poor. The methods above just consider the situation when indexes are multiple and quantified uneasily. However, both the methods are not appropriate choose aero-engine design integration system key influencing factors problem.

The grey correlation analysis [5] of the grey-system theory is an appropriate method to predict things' tendency. Based on the comparability and similarity of orders of number, it analyzes the relevant degree of the main factors inside the system, which helps to define more relevant factors. While the data is given, the grey correlation analysis method could define factors which have more effect on the investigated subject. Therefore, it can be applied to choose aero-engine design integration system key influencing factors by analyzing the relation data [6]-[7]. Compared to the frequently adopted grey correlation calculation based on mean value, which may possibly dwarf the individual information of relevant factors, the grey correlation based on the norm concept is more direct in integrity, thus can be fully applied to calculate the grey correlation and make the correlation analysis quantified.

\section{The grey correlation analysis of the norm}

\subsection{Section headings}

Suppose: $\quad X_{0}=\left\{X_{0}(1), X_{0}(2), \ldots, X_{0}(\mathrm{n})\right\} \quad$ is reference order, namely $X_{0}=\left\{X_{0}(1), X_{0}(2), \cdots, X_{0}(n)\right\} ; X_{j}$ is the comparison order compared with $X_{0}$, namely 
$X_{j}=\left\{X_{j}(1), X_{j}(2), \cdots, X_{j}(n)\right\}(j=1,2, \cdots, m) ; r_{j}(k)$ is the relative D-value between element $K$ in the comparison order $X_{j}$ and the corresponding element in the reference order $X_{0}$, named $r_{j}(k)$ is the grey correlation coefficient of point $k$ which $X_{j}$ relates to $X_{0} ; r_{j}$ is the degree of grey correlation which the comparison order $X_{j}$ corresponds to the reference order $X_{0}=\left\{X_{0}(1), X_{0}(2), \ldots, X_{0}(\mathrm{n})\right\}$ [8].

$$
\begin{gathered}
r_{j}(k)=\frac{\min _{j} \min _{k}|A|+\rho \max _{j} \max _{k}|A|}{|A|+\rho \max _{j} \max _{k}|A|} \\
r_{j}=\frac{1}{n} \sum_{k=1}^{n} r_{j}(k)
\end{gathered}
$$

Among which, $A=x_{0}(k)-x_{j}(k) \cdot \rho$ is recognition coefficient to improve the significant variance between correlation coefficients. $\eta_{j}$ is the sequence of number which makes up with grey correlation coefficient of all points in comparison order $j, \eta_{j}=\left\{r_{j}(k) / k=1,2, \cdots, n\right\}$. Then

$$
\eta^{+}=\left\{\max _{j} r_{j}(k) / j=1,2, \ldots, m ; k=1,2, \ldots, n\right\}
$$

$=\left\{\eta^{+}(k) / k=1,2, \cdots, n\right\}$ is defined as the positive ideal array;

$$
\eta^{-}=\left\{\min _{j} r_{j}(k) / j=1,2, \ldots, m ; k=1,2, \ldots, n\right\}
$$

$=\left\{\eta^{-}(k) / k=1,2, \cdots, n\right\}$ is defined as the negative ideal array. $\eta^{+}$is used to describe the nearest comparison array from reference array, $\eta^{-}$is used to describe the farthest comparison array from reference array.

\subsection{The definition of the grey correlation of the norm}

The two definitions of the norm for the correlation coefficient of the $j$ th comparison order $X_{j}(j=1,2, \cdots, m)$ are [9]:

$$
\begin{aligned}
& d_{j}^{+}=\sqrt{\sum_{k=1}^{n}\left(\eta_{j}(k)-\eta^{+}(k)\right)^{2}}(k=1,2, \cdots, n) \\
& d_{j}^{-}=\sqrt{\sum_{k=1}^{n}\left(\eta_{j}(k)-\eta^{-}(k)\right)^{2}}(k=1,2, \cdots, n)
\end{aligned}
$$

Among which, $d_{j}^{+}$is the near distance of comparison array $j, d_{j}^{-}$is the remote distance of comparison array $j$. During the process to defining correlation array, near distance is smaller, remote distance is larger, and then the relevance of comparison array is stronger.

The grey correlation of the norm is defined as:

$$
\varepsilon_{j}=d_{j}^{-} / d_{j}^{+}+d_{j}^{-}(j=1,2, \cdots, m)
$$

Among which, $\varepsilon_{j}$ is the grey correlation degree of the comparison array $j$. If $d_{j}^{-}=0$, then $\varepsilon_{j}=0$, vice versa, the comparison array at this time is the weakest correlation array; if $d_{j}^{+}=0$, then $\varepsilon_{j}=+\infty$, vice versa, the comparison array at this time is the strongest correlation array.

Compared to the traditional grey correlation, the grey correlation of the norm has more advantages. The traditional grey correlation may possibly dwarf the individual information of relative factors, which would lead to the misjudgment about the ultimate correlation sequence. This is opposite to the goal of property in the grey system information conversion. The grey correlation of the norm may re-excavate the individual information without changing the correlation coefficient. It is a complementary process of information. Therefore, the grey correlation of the norm is an improvement on the traditional grey correlation. Its application in the grey correlation analysis could check the correctness of results further.

\section{Application in identify key factors of aero-engine design integration system}

The aeroengine design integration system has been built since 2011. By 2017, the main functions of the system cover eight aspects, which are also the eight influence factors of the aero engine design integration system: process management (using A instead), tools integration (using B instead), data management (using C instead), standard specification management (using D instead), knowledge management (using $\mathrm{E}$ instead), system management (using $\mathrm{F}$ instead), confidential management (using $\mathrm{G}$ instead) and interface with other systems(using $\mathrm{H}$ instead). A total of 167 designers from 26 related

\begin{tabular}{|c|c|c|c|c|}
\hline Item & 2011 & 2012 & 2013 & 2014 \\
\hline $\mathrm{A}$ & 75524 & 80944 & 87655 & 104651 \\
\hline B & 11050 & 11599 & 12293 & 12945 \\
\hline $\mathrm{C}$ & 28323 & 30501 & 27241 & 27710 \\
\hline $\mathrm{D}$ & 15621 & 11459 & 12123 & 12728 \\
\hline $\mathrm{E}$ & 13954 & 16757 & 19083 & 20818 \\
\hline $\mathrm{F}$ & 18968 & 20860 & 24118 & 31933 \\
\hline G & 41903 & 46015 & 48110 & 45323 \\
\hline $\mathrm{H}$ & 65230 & 67294 & 75220 & 86631 \\
\hline Item & & & 2016 & 2017 \\
\hline
\end{tabular}
departments participated in the judgment, as tab.1 shows, if comparison order and reference order change correspondingly, we can say they have positive correlation. The closer positive changing trend is, the larger correlation is, vice versa.

Table 1. Primal data from 2011 to 2017. 


\begin{tabular}{cccc}
\hline A & 112520 & 129546 & 129094 \\
\hline B & 13956 & 15965 & 19817 \\
\hline C & 28611 & 18510 & 19422 \\
\hline D & 11627 & 11620 & 10833 \\
\hline E & 24137 & 21895 & 23308 \\
\hline F & 29132 & 24895 & 29898 \\
\hline G & 48561 & 54752 & 64790 \\
\hline H & 100524 & 117112 & 128219 \\
\hline
\end{tabular}

As there are some data with great deviation, we need to carry on preliminary treatment on these data. Firstly, we could deal with abnormal values to make the historical data order change more smoothly. As for a group of data $x_{1}, \cdots, x_{n}$, name $\bar{x}=\frac{1}{n} \sum_{i=1}^{n} x_{i}$, if $x_{i}>\bar{x}(1+20 \%)$, then $x_{i}=\bar{x}(1+20 \%)$; if $x_{i}<\bar{x}(1-20 \%)$, then $x_{i}=\bar{x}(1-20 \%)$.

Secondly we could use dynamic irregular curve to describe the variation of the data distinctly. Because both the dimension of primary data and the numerical gradation have sharp differences, we need deal with primary data in advance to gain the comparativeness among data. Data are normalized according to the principle of $x_{j}^{\prime}(k)=x_{j}(k) / x_{j}(1), j=1,2, \cdots, m, k=1,2, \cdots, n$ Results are shown in tab.2.

Table 2. Processed data by standardization.

\begin{tabular}{cccc}
\hline $\mathrm{A}$ & $\mathrm{B}$ & $\mathrm{C}$ & $\mathrm{D}$ \\
\hline 1 & 1 & 1 & 1 \\
\hline 1.0718 & 1.0496 & 1.0770 & 0.7336 \\
\hline 1.1606 & 1.1124 & 0.9619 & 0.7761 \\
\hline 1.3857 & 1.1714 & 0.9785 & 0.8148 \\
\hline 1.4899 & 1.2630 & 1.0102 & 0.7443 \\
\hline 1.7153 & 1.4447 & 0.6536 & 0.7439 \\
\hline 1.7093 & 1.7933 & 0.6857 & 0.6935 \\
\hline $\mathrm{E}$ & $\mathrm{F}$ & $\mathrm{G}$ & $\mathrm{H}$ \\
\hline 1 & 1 & 1 & 1 \\
\hline 1.2009 & 1.0977 & 1.0981 & 1.0316 \\
\hline 1.3676 & 1.2715 & 1.1481 & 1.1532 \\
\hline 1.4919 & 1.6835 & 1.0816 & 1.3281 \\
\hline 1.7298 & 1.5358 & 1.1589 & 1.5411 \\
\hline 1.5691 & 1.3125 & 1.3066 & 1.7954 \\
\hline 1.6703 & 1.5762 & 1.5462 & 1.9656 \\
\hline
\end{tabular}

Select $\rho=0.5$, correlation coefficients are figured out according to formula (1), results are shown in tab.3.

Table 3. Correlation coefficients.

\begin{tabular}{cccc}
\hline $\mathrm{A}$ & $\mathrm{B}$ & $\mathrm{C}$ & $\mathrm{D}$ \\
\hline 1 & 1 & 1 & 1 \\
\hline 0.9604 & 0.9990 & 0.9514 & 0.6233 \\
\hline 0.9263 & 0.9878 & 0.7694 & 0.6045 \\
\hline 0.7326 & 0.9581 & 0.7082 & 0.5799 \\
\hline 0.7221 & 0.9541 & 0.6534 & 0.4907 \\
\hline 0.6745 & 0.9674 & 0.3932 & 0.4217 \\
\hline 0.8966 & 0.9569 & 0.3259 & 0.3275 \\
\hline
\end{tabular}

\begin{tabular}{cccc}
\hline $\mathrm{E}$ & $\mathrm{F}$ & $\mathrm{G}$ & $\mathrm{H}$ \\
\hline 1 & 1 & 1 & 1 \\
\hline 0.7767 & 0.9136 & 0.9162 & 0.9658 \\
\hline 0.6782 & 0.7745 & 0.9472 & 0.9387 \\
\hline 0.6378 & 0.5172 & 0.8229 & 0.7967 \\
\hline 0.5427 & 0.6791 & 0.8021 & 0.6745 \\
\hline 0.8308 & 0.7775 & 0.7709 & 0.6115 \\
\hline 0.8406 & 0.7303 & 0.7010 & 0.7279 \\
\hline
\end{tabular}

According to formula (2), the grey correlations are worked out. Tab.4 lists results in accordance with the order of the grey correlations. The results of tal.4 are calculated by the traditional grey correlation analysis based on average values. According to the correlation coefficient of tab.3, the positive ideal array is defined as:

$$
\eta^{+}=\{1,0.9990,0.9878,0.9581,0.9541,0.9674,0.9569\}
$$

The negative ideal array is defined as:

$$
\eta^{-}=\{1,0.6233,0.6045,0.5172,0.4907,0.3932,0.3259\}
$$

According to formula (3) and (4), the near distances and far distances of each comparison column are worked out, referring to tab.4.

Table 4. Near distance and far distance of each parameter.

\begin{tabular}{ccccc}
\hline Parameter & $\mathrm{A}$ & $\mathrm{B}$ & $\mathrm{C}$ & $\mathrm{D}$ \\
\hline Near distance & 0.4465 & 0 & 0.9647 & 1.1575 \\
\hline Far distance & 0.8497 & 1.1938 & 0.4447 & 0.0689 \\
\hline \multicolumn{1}{c}{} & & & \\
\hline Parameter & $\mathrm{E}$ & $\mathrm{F}$ & $\mathrm{G}$ & $\mathrm{H}$ \\
\hline Near distance & 0.6703 & 0.6405 & 0.3924 & 0.5356 \\
\hline Far distance & 0.7090 & 0.6782 & 0.8228 & 0.7417 \\
\hline
\end{tabular}

Table 5. The grey correlation of each parameter.

\begin{tabular}{ccccc}
\hline Parameter & $\mathrm{B}$ & $\mathrm{G}$ & $\mathrm{A}$ & $\mathrm{H}$ \\
\hline The grey correlation & 0.975 & 0.851 & 0.845 & 0.816 \\
\hline \multicolumn{6}{c}{} & & & & \\
\hline Parameter & $\mathrm{F}$ & $\mathrm{E}$ & $\mathrm{C}$ & $\mathrm{D}$ \\
\hline The grey correlation & 0.770 & 0.758 & 0.686 & 0.578 \\
\hline
\end{tabular}

Table 6. The grey correlation based on normal number of each parameter

\begin{tabular}{cccccc}
\hline Parameter & 1 & 2 & 3 & 4 \\
\cline { 2 - 5 } & $\mathrm{B}$ & $\mathrm{G}$ & $\mathrm{A}$ & $\mathrm{H}$ \\
\hline $\begin{array}{c}\text { The grey correlation } \\
\text { based on norm }\end{array}$ & $+\infty$ & 2.9196 & 2.7526 & 2.1266 \\
\hline & & & & \\
\hline Parameter & 5 & 6 & $\mathrm{E}$ & $\mathrm{C}$ & $\mathrm{D}$ \\
\hline & 1.7666 & 1.7370 & 0.9057 & 0.1284 \\
\hline $\begin{array}{c}\text { The grey correlation } \\
\text { based on norm }\end{array}$ & &
\end{tabular}

Comparing tab.4 with tab. 6 , we can see that the results are generally similar. However, there is still a little difference about correlations degree sequence. Referring 
to the results, the order of the grey norm correlation is closer to the actual situation. The results indicate that the application of the grey correlation analysis of the norm improve the accuracy of the traditional grey correlation method. The grey correlation can quantitatively define the impact that comparison orders have on the reference order by dealing with data. But traditional grey correlation analysis may dwarf individual information of relevant factors. The grey correlation of the norm may reexcavate the individual information, which can avoid misjudgments of the correlation sequence. The correlation degree of the eight influencing factors was identified effectively, which provided the direction for the further improvement and optimization of the aeroengine design integration system.

\section{Acknowledgement}

This research was financially supported by the Humanity and Social Science Youth foundation of Ministry of Education (No.12YJC790229).

\section{References}

1. $\mathrm{Hu}$ Yonghong, He Sihui. Comprehensive evaluation method. Beijing: China science press, 2000.

2. Tian Y, Zhu D. A Research on Structure of Logistics Alliance [J]. Logistics Technology, 2000, 2: 012.
3. Feng $\mathrm{S}, \mathrm{Xu}$ L D. Decision support for fuzzy comprehensive evaluation of urban development $[\mathrm{J}]$. Fuzzy Sets \& Systems, 1999, 105 (97): 1-12.

4. Li L, Shen L. An improved multilevel fuzzy comprehensive evaluation algorithm for security performance $[\mathrm{J}]$. The Journal of China Universities of Posts and Telecommunications, 2006, 13 (4): 4853.

5. Zhang J, Wu D, Olson D L. The method of grey related analysis to multiple attribute decision making problems with interval numbers [J]. Mathematical and computer modelling, 2005, 42 (9): 991-998.

6. Liu W W, Shi C S, Zhao S B. Dynamic comprehensive evaluation model with the feature of speed $[\mathrm{J}]$. Systems Engineering-Theory \& Practice, 2013.

7. Wei C, Liu P, Chang C. Failure mode and effects analysis using grey theory $[\mathrm{J}]$. Integrated Manufacturing Systems, 1990, volume 12 (3): 211216 (6).

8. Deng Julong. The basis of grey theory. Wuhan: Huazhong University of Science \&Technology press, 2002.

9. Zhang Zhilong, Wang Lansheng. Application of the grey correlation analysis of the norm in the grouting reinforcement project. Journal of engineering geology, 2005, 13 (1): 140-143. 